\title{
Prophylactic Central Neck Dissection in the Management of Papillary Thyroid Carcinoma; is there a Meaningful Outcome?
}

\author{
*Sohail Bakkar \\ University Hospital of Pisa, Department of Surgical Pathology, Italy \\ Submission: May 28 2016; Published: June 17, 2016 \\ *Corresponding author: Sohail Bakkar, University Hospital of Pisa, Department of Surgical Pathology, Division of Endocrine Surgery, Via \\ Paradisa2, 56124 Pisa, Italy, Tel: +393441162487; Fax: +39050997709; Email: sohail.bakkar@gmail.com
}

\begin{abstract}
Papillary thyroid carcinomas are favorable prognosis cancers. Most patients have an excellent long term overall survival rate. As a result, disease-free survival has replaced overall survival as the main outcome of interest when effective initial management of these tumors is considered. The role of prophylactic central neck dissection represents an area of ongoing controversy in this regards. Our groups' opinion is that in the context of available literature, performing central neck dissection routinely fails to provide a clinically meaningful outcome and only condemns patients to its potential serious sequelae.
\end{abstract}

Keywords: Papillary thyroid cancer; Prophylactic; Routine; Central neck dissection

Abbreviations: PTC: Papillary thyroid carcinoma; pCCND: Prophylactic central compartment neck dissection; RAI: Radioactive iodine; AJCC: American Joint Committee on Cancer; TNM: Tumor, nodal disease and distant metastasis

\section{Introduction}

Thyroid cancer has the highest increase in incidence rate among all cancers, and this is largely but not full attributed to the increased detection of subclinical papillary carcinomas [1]. As a result, the surgical strategy undertaken in the management of these tumors has been gaining increasing popularity among Endocrinologists and Endocrine Surgeons. Papillary thyroid carcinomas (PTC) are considered favorable prognosis cancers. Most patients have a 5-year overall survival rate well above $90 \%$ [2]. Therefore, disease-free survival has replaced overall survival as the main outcome of interest when effective initial management of these tumors is evaluated. Given the high incidence of occult nodal metastasis in PTC, advocating prophylactic central compartment neck dissection (pCCND) for the initial management of these tumors is, in theory, the ideal thing to do. Nevertheless, the role of pCCND in the management of PTC remains controversial regarding its benefits and risks [3-6].

Prophylactic neck dissection is defined as neck dissection in the absence of clinical evidence of nodal involvement whether on preoperative examination and imaging studies or during intraoperative evaluation. It is synonymous with elective/routine dissection [7].
Essential to any assessment of the role of pCCND is to verify its oncologic rationale and whether its benefit outweighs its potential morbidity. Proposed potential benefits of pCCND include: eliminating a potential source of recurrence subsequently avoiding potential morbidity of revision surgery, increasing the accuracy of disease staging for radioactive iodine (RAI) dosing and long-term follow up, and improved accuracy of thyroglobulin surveillance [6]. While occult node positivity is quite common in PTC (even in subcentimetric cancers), it rarely demonstrates prognostic parameters of recurrence and/or a possibly worse diseasespecific survival. Occult metastases usually tend to be small in size and number, with no extranodal extension, and a median risk of recurrence of only $2 \%$ [8]. Moreover, the comparability in oncologic outcome between those who undergo pCCND and those who do not is a testimony to the indolent biologic behavior of subclinical nodal disease [9-14]. Further proof of the clinical stability of occult nodal disease, is the rare progression to locoregional recurrence among untreated patients [15] or those who did not receive RAI adjuvant therapy [16]. A recent meta-analysis demonstrated that the presence of occult central nodal metastasis was not a significant predictor of recurrence nor did pCCND improve local control [17]. Reoperation for recurrent disease in the central neck, despite being uncommon, is challenging. 
However, in experienced hands the safety of CCND as a secondary operation was comparable to it as a primary one $[18,19]$.

One of the arguments for the use of pCCND is that it influences the usage of RAI through identifying the true nodal status of patients. Accordingly patients may be stratified to receive higher doses of RAI [13] or spared empiric postoperative RAI therapy [20]. The American joint committee on cancer (AJCC) tumor, nodal disease and distant metastasis (TNM) staging system considers nodal metastasis a prognostic factor for patients above the age of 45 years [21]. However, it does not differentiate between microscopic and macroscopic metastases that have different implications on outcome. Therefore, microscopic upstaging may lead to potentially unnecessary or additional treatments and reevaluations that are not devoid of adverse consequences [22]. On the other hand, giving empiric postoperative RAI based on the assumption of node positivity does not seem to be appropriate especially that the clinical stability of occult metastases in untreated patients or those who did not receive postoperative RAI has been demonstrated $[15,16]$.

Performing pCCND is in theory a step forward towards achieving athyroglobulinemia and improving surveillance accuracy. Nevertheless, the literature has been inconsistent in demonstrating this $[5,13,23]$. Furthermore, factors other than the normalization of serum thyroglobulin levels play a more important role in modifying follow-up management [2]. As far as morbidity of pCCND is concerned it has been demonstrated that studies comparing total thyroidectomy alone to that with pCCND, show a significant increase in transient hypoparathyroidism and a trend towards increased permanent hypoparathyroidism and RLN injury with the latter [2]. Therefore, it seems fair to say that since pCCND does not have a favorable prognostic impact (in terms of both disease-specific and disease-free survival), and is associated with an increased risk of postoperative morbidity avoiding the procedure in clinically node negative patients would translate into preservation of their quality of life following surgery. This in turn is considered an extremely meaningful outcome particularly in oncologic patients, and has been demonstrated in the first prospective randomized controlled trial on the role of pCCND in the management of PTC in literature conducted at the author's centre.

As the vast majority of PTCs are associated with nodal micrometastasis studies focusing on clinical predictors of recurrence rather than nodal metastasis occurrence are required to guide the selection of potential subgroups of patients in which pCCND could be recommended. In conclusion, the low recurrence rate in the central neck combined with the potential for increased morbidity from pCCND or the need for RAI or its higher doses as a result of microscopic upstaging of tumors outweighs the potential benefits of routinely performing the procedure. Therefore, thyroidectomy without pCCND seems to be appropriate for patients with PTC in the absence of clinically apparent metastatic nodes.

\section{Disclosure}

The author has no financial ties or conflicts of interest to disclose.

\section{Reference}

1. Morris L, Myssiorek D (2010) Improved detection does not fully explain the rising incidence of well-differentiated thyroid cancer: a population-based analysis. Am J Surg 200(4): 454-461.

2. Gyorki DE, Untch B, Tuttle RM, Shaha AR (2013) Prophylactic central neck dissection in differentiated thyroid cancer: an assessment of the evidence. Ann Surg Oncol 20(7): 2285-2289.

3. Bardet S, Ciappuccini R, Quak E, Rame JP, Blanchard D, et al. (2015) Prognostic value of microscopic lymph node involvement in patients with papillary thyroid cancer. J Clin Endocrinol Metab 100(1): 132140 .

4. Hartl DM, Mamelle E, Borget I, Leboulleux S, Mirghani H, et al. (2013) Influence of prophylactic neck dissection on rate of retreatment for papillary thyroid carcinoma. World J Surg 37(8): 1951-1958.

5. Barczyński M, Konturek A, Stopa M, Nowak W (2013) Prophylactic central neck dissection for papillary thyroid cancer. Br J Surg 100(3): 410-418.

6. Cranshaw IM, Carnaille B (2008) Micrometastases in thyroid cancer. An important finding? Surg Oncol 17(3): 253-258.

7. American Thyroid Association Surgery Working Group; American Association of Endocrine Surgeons; American Academy of Otolaryngology-Head and Neck Surgery; American Head and Neck Society, Carty SE, et al. (2009) Consensus statement on the terminology and classification of central neck dissection for thyroid cancer. Thyroid 19(11): 1153-1158.

8. Randolph GW, Duh QY, Heller KS, LiVolsi VA, Mandel SJ, et al. (2012) The prognostic significance of nodal metastases from papillary thyroid carcinoma can be stratified based on the size and number of metastatic lymph nodes, as well as the presence of extranodal extension. Thyroid 22(11): 1144-1152.

9. Viola D, Materazzi G, Valerio L, Molinaro E, Agate L, et al. (2015) Prophylactic central compartment lymph node dissection in papillary thyroid carcinoma: clinical implications derived from the first prospective randomized controlled single institution study. J Clin Endocrinol Metab 100(4): 1316-1324.

10. Conzo G, Calò PG, Sinisi AA, De Bellis A, Pasquali D, et al. (2014) Impact of prophylactic central compartment neck dissection on locoregional recurrence of differentiated thyroid cancer in clinically node-negative patients: a retrospective study of a large clinical series. Surgery 155(6): 998-1005.

11. Ywata de Carvalho A, Chulam TC, Kowalski LP (2015) Long-term results of observation vs. prophylactic selective level VI neck dissection for papillary thyroid carcinoma at a cancer center. JAMA Otolaryngol Head Neck Surg 141(7): 599-606.

12. Liu J, Xu Z, Li Z, Zhang Z, Tang P, et al. (2015) Long-term outcomes of observation for clinically negative central compartment lymph nodes in papillary thyroid carcinoma. Eur Arch Otorhinolaryngol 272(12): 3801-3804.

13. Hughes DT, White ML, Miller BS, Gauger PG, Burney RE, et al. (2010) Influence of prophylactic central lymph node dissection on postoperative thyroglobulin levels and radioiodine treatment in papillary thyroid cancer. Surgery 148(6): 1100-1106.

14. Moo TA, McGill J, Allendorf J, Lee J, Fahey T, et al. (2010) Impact of prophylactic central neck lymph node dissection on early recurrence in papillary thyroid carcinoma. World J Surg 34(6): 1187-1191. 


\section{Open Access Journal of Surgery}

15. Ito Y, Miyauchi A, Inoue H, Fukushima M, Kihara M, et al. (2010) An observational trial for papillary thyroid microcarcinoma in Japanese patients. World J Surg 34(1): 28-35.

16. Wada N, Duh QY, Sugino K, Iwasaki H, Kameyama K, et al. (2003) Lymph node metastasis from 259 papillary thyroid microcarcinomas: frequency, pattern of occurrence and recurrence, and optimal strategy for neck dissection. Ann Surg 237(3): 399-407.

17. Qu N, Zhang L, Ji QH, Chen JY, Zhu YX, et al. (2015) Risk factors for central compartment lymph node metastasis in papillary thyroid microcarcinoma: a meta-analysis. World J Surg 39(10): 2459-2470.

18. Alvarado R, Sywak MS, Delbridge L, Sidhu SB (2009) Central lymph node dissection as a secondary procedure for papillary thyroid cancer: Is there added morbidity? Surgery 145(5): 514-518.

19. Shen WT, Ogawa L, Ruan D, Suh I, Kebebew E, et al. (2010) Central neck lymph node dissection for papillary thyroid cancer: comparison of complication and recurrence rates in 295 initial dissections and reoperations. Arch Surg 145(3): 272-275.
20. Ruel E, Thomas S, Dinan MA, Perkins JM, Roman SA, et al. (2015) Knowledge of pathologically versus clinically negative lymph nodes is associated with reduced use of radioactive iodine post-thyroidectomy for low-risk papillary thyroid cancer. Endocrine 52(3): 579-586.

21. Edge SB, Compton CC (2010) AJCC Cancer Staging Manual, 7th edition. Ann Surg Oncol 17(6): 1471-1474.

22. Clement SC, Peeters RP, Ronckers CM, Links TP, van den Heuvel-Eibrink $\mathrm{MM}$, et al. (2015) Intermediate and long-term adverse effects of radioiodine therapy for differentiated thyroid carcinoma-A systematic review. Cancer Treat Rev 41(10): 925-934.

23. Popadich A, Levin O, Lee JC, Smooke-Praw S, Ro K, et al. (2011) A multicenter cohort study of total thyroidectomy and routine central lymph node dissection for cN0 papillary thyroid cancer. Surgery 150(6): 1048-1057. 